\title{
Entre unité de soins intensifs et unité de lits «standard»
}

\author{
Luca Lavina ${ }^{a}$, Michael Wehrlib, Thierry Fumeaux ${ }^{c}$ \\ a Secrétariat général SSMI; ${ }^{b}$ Président gestionnaire SSMI; ${ }^{c}$ Prof., président médecins SSMI, membre FMH
}

Durant un séjour stationnaire à l'hôpital, les patients doivent être traités de la manière la plus compétente, au bon moment et au bon endroit. Où est la place des patients qui sont trop malades pour être pris en charge dans un service normal, mais qui en même temps ne nécessitent pas les mesures complexes de la médecine intensive? Cette question est pendant longtemps restée sans réponse claire et les patients étaient souvent traités dans des services qui n’avaient pas été conçus pour leur situation clinique. Toutefois, depuis tout juste 20 ans, on assiste à la création, dans les hôpitaux suisses de services qui sont précisément spécialisés dans le traitement de ces patients, dans le but de combler cet espace lacunaire entre unité de lits et unité de soins intensifs (USI). Depuis l'automne dernier, ces unités de soins intermédiaires (en abrégé U-IMC) sont visitées et reconnues par un groupe de travail interdisciplinaire et interprofessionnel, sous l'égide de la Société Suisse de Médecine Intensive (SSMI). La SSMI étant convaincue de la place primordiale des U-IMC dans le système de santé suisse, elle en a fait le thème principal du SSMI Symposium de cette année, qui s'est tenu début mars à l'Inselspital de Berne.

\section{Reconnaissance officielle des U-IMC}

L'histoire des U-IMC a débuté vers la fin des années 1960. Les patients victimes d'infarctus du myocarde et présentant un risque accru de complications étaient regroupés dans des Coronary Care Units (unités de soins coronaires), où ils bénéficiaient d'une prise en charge globale par du personnel spécialisé, permettant de réduire considérablement la mortalité de ce groupe de patients.

Sur le modèle des Coronary Care Units, des U-IMC ont commencé à voir le jour en Suisse au cours des années 1990. Toutefois, ce n'est qu'à partir de 2011 que neuf sociétés de discipline médicale et infirmière suisses ont élaboré, sous l'impulsion initiale de la SSMI, des directives spécifiques pour les U-IMC, dans le but de garantir la qualité des soins dans ces services. Depuis l'été dernier, les U-IMC peuvent demander une reconnaissance officielle par une commission ad hoc, et ainsi facturer leurs prestations dans des DRG spécifiquement prévus à cet effet, précise Constanze Hergeth de SwissDRG. Comme le souligne le Dr Jolanda Contartese, cheffe de l'U-IMC de l'Hôpital cantonal de Baden et vice-présidente de la Commission pour la reconnaissance des unités de soins intermédiaires (CRUIMC), la plupart des U-IMC de Suisse se consacrent à une typologie de patients particulière ou sont rattachées à une discipline médicale, comme les unités de soins coronaires. Il existe toutefois aussi en Suisse plusieurs U-IMC générales ou interdisciplinaires sans spécialisation particulière. Les U-IMC peuvent fonctionner de manière indépendante ou être rattachées à d'autres services (le plus souvent une USI) situés dans le même hôpital, voire dans un autre hôpital.

\section{Flexibilité et diversité}

L'offre thérapeutique et la relation avec les autres services et cliniques dépendent en grande partie des missions de l'U-IMC, des professionnels qui y travaillent, de l'hôpital et de la typologie des patients admis. Les directives pour la reconnaissance des U-IMC autorisent une certaine flexibilité et par conséquent une grande diversité de ces services, ce qui constitue précisément l'une de leurs grandes forces. D'après Marco Maggiorini, ancien président de la SSMI, cela donne également la possibilité à de plus petites institutions, pour lesquelles l'exploitation d'une USI est trop coûteuse, de mettre en place une U-IMC. A cet effet, ces institutions doivent toutefois établir une coopération avec un hôpital doté d'une USI.

Toutes les U-IMC ont comme point commun de traiter des patients ayant un besoin important de soins. Dans une USI, un infirmier ou une infirmière s'occupe d'un à deux patients; dans un service normal, un infirmier ou une infirmière s'occupe de jusqu'à huit patients (jusqu'à 24 durant la nuit). L'U-IMC se trouve entre les deux, avec trois à quatre patients par infirmier ou infirmière. Il existe trois trajectoires différentes qui mènent ces patients à une U-IMC, explique Elisabeth 
Allemann de l'Inselspital de Berne. Certains patients sont transférés dans une U-IMC après des opérations; d'autres y sont amenés en urgence, souvent aussi parce que la surveillance dans l'unité de lits n'est plus suffisante et doit être renforcée. Dans ces cas, l'U-IMC correspond à une Step-Up-Unit. Lorsqu'un patient est transféré de l'USI à l'U-IMC après la stabilisation des paramètres vitaux, il s'agit alors plutôt d'une Step-DownUnit. Le transfert de l'U-IMC dans d'autres services fonctionne selon le même principe. Lorsqu'un patient va mieux, il est transféré dans une unité de lits. Si l'état de santé d'un patient se détériore, par ex. en raison d'une défaillance aiguë de plus d'un organe, on procède à nouveau à un Step-Up de l'U-IMC vers l'USI.

\section{Une prise en charge médicale sur mesure}

Ainsi, les U-IMC peuvent offrir une prise en charge médicale sur mesure à une variété de patients présentant des affections et antécédents médicaux divers, et de cette manière, ce qui constitue une évolution du concept thérapeutique à deux niveaux de soins d'un hôpital vers un concept à trois niveaux. Cela augmente l'efficience des USI et des services généraux, qui peuvent ainsi traiter les patients pour lesquels ils sont réellement conçus, en concordance avec leur mission.

Comme dans les USI, le travail dans les U-IMC est profondément marqué par l'interprofessionnalité et par l'interdisciplinarité. Les médecins et les soignants, mais également les thérapeutes de différentes disciplinaires, y travaillent en étroite collaboration, ce qui, d'après Martine Louis Simonet des HUG de Genève, génère de nouvelles connaissances et profite au final au patient. Les deux groupes professionnels estiment qu'une formation postgraduée dans le domaine des soins intermédiaires est nécessaire à la fois pour les soignants et les médecins: pour Thierry Fumeaux, président médecins de la SSMI, une spécialisation du personnel médical d'une U-IMC est tout à fait concevable à l'avenir. Hans Richter, responsable de la formation professionnelle Soins intensifs EPD ES, est également d'avis que les soins dispensés en U-IMC requièrent des compétences spécifiques et qu'une formation postgraduée pratique dans ce domaine précis revêt une grande importance. Actuellement, des exigences minimales pour une formation postgraduée homogène en soins intermédiaires des infirmiers diplômés sont élaborées à l'échelle nationale par l'OdASanté.

Tout comme au sein de l'équipe thérapeutique dans l'U-IMC, l'interprofessionnalité et l'interdisciplinarité sont également essentielles au sein de la CRUIMC. La commission est désormais composée de plus de 30 re- présentants engagés (médecins et soignants) de la médecine intensive, de l'anesthésiologie et de la réanimation, de la cardiologie, de la chirurgie, de la chirurgie pédiatrique, de la médecine interne générale, de la pédiatrie, des neurosciences cliniques et de la néonatalogie. Sans la collaboration interprofessionnelle et interdisciplinaire entre les membres de cette commission, la reconnaissance des U-IMC serait tout bonnement impossible.

Malgré le fait que les participants au symposium s'accordent sur le principe de l'U-IMC comme service interfacé entre les deux autres niveaux de soins et adhèrent aux critères de qualité élaborés par la commission, certains soulignent la nécessité d'une révision des directives. Tandis que Peter Altherr, responsable de l'Office de la Santé en charge de l'organisation des soins médicaux (Amt für Gesundheitsversorgung) du canton de Saint-Gall, a émis le souhait d'une meilleure connexion entre les directives sur les U-IMC et les exigences cantonales en matière de planification hospitalière, Jürg

\section{Comme dans les USI, le travail dans les U-IMC} est profondément marqué par l'interprofessionnalité et par l'interdisciplinarité.

Hodler, directeur médical de l'Hôpital universitaire de Zurich, a quant à lui estimé que les 20 pages de directives contenaient trop de prescriptions relatives aux locaux et à l'architecture, alors que les exigences médicales sont au contraire abordées trop sommairement. En réponse, Jolanda Contartese souligne que beaucoup de critères relatifs à l'architecture sont des critères qui ne sont pas tous nécessaires, seuls $60 \%$ devant être remplis pour obtenir la reconnaissance en tant qu'U-IMC, conférant précisément une certaine flexibilité aux critères de qualité. Elle ajoute: «Nous souhaitons donner à de nombreuses U-IMC de Suisse l'opportunité de se faire reconnaître, car notre objectif principal est d'augmenter la qualité des traitements dans les soins intermédiaires, ainsi que de donner la possibilité au personnel de suivre une formation postgraduée dans ce domaine.» Ces suggestions ne semblent modifier en rien le vif intérêt que suscite la reconnaissance des UIMC: à la fin du mois d'avril 2017, 22 U-IMC avaient déjà été reconnues par la CRUIMC et neuf autres services avaient sollicité une reconnaissance dans cette optique, avec une tendance à la hausse.

Vous pouvez visualiser toutes les présentations du SSMI Symposium 2017 sous forme de Webcasting sur www.sgi-ssmi.ch. Des informations supplémentaires sur les soins intermédiaires sont disponibles sur www.swiss-imc.ch. 